\title{
ON STANDARD YOUNG TABLEAUX OF BOUNDED HEIGHT
}

\author{
M J MISHNA
}

\begin{abstract}
We survey some recent works on standard Young tableaux of bounded height. We focus on
\end{abstract} consequences resulting from numerous bijections to lattice walks in Weyl chambers.

\section{INTRODUCTION}

Standard Young tableaux are a classic object of mathematics, appearing in problems from representation theory to bijective combinatorics. Lattice walks restricted to cones are similarly a fundamental family, and they encode a wide variety of combinatorial structures from formal languages to queues. Standard Young tableaux of bounded height are in bijection with several different straightforward classes of lattice walks. This connection not only elucidates several sources of ubiquity on both accounts, but facilitates exact and asymptotic enumeration, as well as parameter analysis. This survey describes the cross developments over the past the past 30 years, and highlights some open problems. For more background on the tableaux, we recommend the surveys [33, 1] and particularly the article of Stanley [36].

We begin by fixing our notation and conventions. Let $\lambda=\left(\lambda_{1}, \lambda_{2}, \ldots, \lambda_{t}\right)$ be a partition of $n$ into $t$ parts. We write $\lambda \vdash n$ and $\ell(\lambda)=t$ in this case. The Ferrers diagram of shape $\lambda$ is a representation of $\lambda$ comprised of boxes indexed by pairs $\left\{(i, j): 1 \leq i \leq t ; 1 \leq j \leq \lambda_{i}\right\}$. Such a diagram is of size $n$, and height $t$. A key parameter in our study is the number of columns of odd length.

A standard Young tableau of size $n$ is a filling of a Ferrers diagram using precisely the integers 1 to $n$. The entries strictly increase to the right along each row and strictly increase down each column. A tableau is semi-standard if the entries weakly increase along each row and strictly increase down each column. In this work we are interested in the number of standard Young tableaux of a given size with the height is bounded by a fixed value.

1.1. Enumeration formulas. Rather classically, the number of standard Young tableaux of shape $\lambda$ is denoted $f^{\lambda}$ and is given by the hook length formula:

$$
f^{\lambda}=\frac{n !}{\prod_{c} h_{c}} \quad \text { where } \quad h_{c}=\lambda_{i}+\operatorname{card}\left\{j: \lambda_{j} \geq i\right\}-i-j+1 .
$$

The following formulation is due to MacMahon:

$$
f^{\lambda}=\left(\lambda_{1}+\cdots+\lambda_{d}\right) ! \operatorname{det}\left(\frac{1}{\left(\lambda_{i}-i+j\right) !}\right)_{1 \leq i, j \leq d} .
$$

The number of standard Young tableaux of height at most $k$ is thus the sum

$$
y_{k}(n) \equiv \sum_{\substack{\lambda \vdash n \\ \ell(\lambda) \leq k}} f^{\lambda} .
$$

The first enumerative formulas for Young tableaux where the height is an explicit consideration appear in the 1960s, when Gordon and Houten studied $k$-rowed plane partitions whose non-zero parts strictly decrease along rows and columns, in addition to some related variants. In their series of Notes on Plane Partitions [22, 23], they give some formulas for the generating functions in terms of infinite products and determinants. Regev [32] first determined exact expressions for $y_{2}(n)$ and $y_{3}(n)$. The formulas are equivalent 


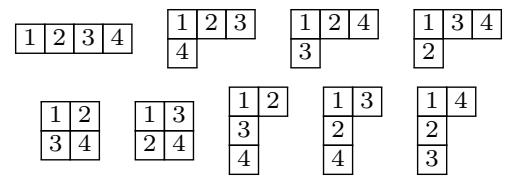

Figure 1. All standard Young tableaux of size 4 and height at most 3

to the following. Here $C_{k}$ denotes the $k$-th Catalan number ${ }^{1}$

$$
y_{2}(n)=\left(\begin{array}{c}
n \\
\lfloor n / 2\rfloor
\end{array}\right) \quad y_{3}(n)=\sum_{k=0}^{\lfloor n / 2\rfloor}\left(\begin{array}{c}
n \\
2 k
\end{array}\right) C_{k} .
$$

The numbers $y_{3}(n)$ are also known as Motzkin numbers. Figure 1 illustrates the nine standard Young tableaux of size four and of height at most three. Around the same time, Gessel [18] found an expression for $y_{4}(n)$, and Gouyou-Beauchamps [25] found the following expressions for $y_{4}(n)$ and $y_{5}(n)$ :

$$
\begin{array}{r}
y_{4}(2 n)=C_{n} C_{n} \quad y_{4}(2 n+1)=C_{n} C_{n+1} \\
y_{5}(n)=\sum_{i=0}^{\lfloor n / 2\rfloor} \frac{3 ! n !(2 i+2) !}{(n-2 i) ! i !(i+1) !(i+2) !(i+3) !} .
\end{array}
$$

No comparable expression for $y_{6}(n)$ has appeared in the literature. The presence of binomials in general, and Catalan numbers in particular, is a strong hint that these tableaux are related to well understood combinatorial classes.

1.2. The exponential generating function. We study $y_{k}(n)$ via $Y_{k}(t)$, the exponential generating function for $y_{k}(n)$ :

$$
Y_{k}(t) \equiv \sum y_{k}(n) \frac{t^{n}}{n !}
$$

The formulas depends on the parity of the height. The formula for $Y_{2 k}(t)$ was obtained by Gordon [21] by reducing a Pfaffian of Gordon and Houten [22, and Gessel [18] found the formula for odd heights. They are both expressed in terms of the hyperbolic Bessel function of the first kind of order $j$

$$
b_{j} \equiv I_{j}(2 t)=\sum_{n=0}^{\infty} \frac{t^{2 n+j}}{n !(n+j) !} .
$$

The formulas are:

$$
\begin{aligned}
Y_{2 k}(t) & =\operatorname{det}\left(b_{i-j}+b_{i+j-1}\right)_{1 \leq i, j \leq k} \\
Y_{2 k+1}(t) & =e^{t} \operatorname{det}\left(b_{i-j}-b_{i+j}\right)_{1 \leq i, j \leq k}
\end{aligned}
$$

For example, $Y_{2}(t)=b_{0}+b_{1}$ and $Y_{4}(t)=b_{0}^{2}+b_{0} b_{1}+b_{0} b_{3}-2 b_{1} b_{2}-b_{2}^{2}-b_{1}^{2}+b_{1} b_{3}$. These expressions grow very fast as polynomials in $b_{j}$, however, they are amenable to some further study including asymptotic analysis of the coefficients. They also imply that $y_{k}(n)$ can be expressed by binomial sums, although an expression that one could compute may be too complex to be of any use.

One important consequence of these formulas is that they resolved a question that Stanley [35] had asked almost 10 years before these formulas appeared about the nature of the function $Y_{k}(t)$. Specifically, he asked if $Y_{k}(t)$ is $D$-finite, that is, does it satisfy a differential equation with polynomial coefficients. GouyouBeauchamps showed that $Y_{4}(t)$ is D-finite from his formula, and Gessel [18] proved this result for general $k$ since the expressions are a polynomial combination of Bessel functions (possibly times an exponential). Bessel functions are D-finite so the result follows from closure properties.

$$
{ }^{1} C_{n} \equiv\left(\begin{array}{c}
2 n \\
n
\end{array}\right) \frac{1}{n+1}
$$


1.3. Schur Functions. To prove these formulas Gessel started with another important formula for standard Young tableaux. Schur functions can be described via a summation over the set of all semi-standard Young tableaux of shape $\lambda$ :

$$
s_{\lambda}=\sum_{T \in \operatorname{SSYT}(\lambda)} x^{\operatorname{content}(T)}=\sum_{T \in \operatorname{SSYT}(\lambda)} x_{1}^{t_{1}} x_{2}^{t_{2}} \ldots x_{k}^{t_{k}} .
$$

The exponents describe the content of the tableau: $t_{i}$ is the number of occurrences of $i$ in $T$. Thus, the coefficient of the monomial $x_{1} \ldots x_{n}$ in this expression is the number of standard Young tableaux of shape $\lambda$. We deduce the formula

$$
y_{k}(n)=\sum_{\substack{\lambda \vdash n \\ \ell(\lambda) \leq k}}\left[x_{1} x_{2} \ldots x_{n}\right] s_{\lambda}=\left[x_{1} x_{2} \ldots x_{n}\right] \sum_{\substack{\lambda \vdash n \\ \ell(\lambda) \leq k}} s_{\lambda} .
$$

This kind of coefficient extraction in symmetric functions can be framed as a homomorphism. This was done by Gessel in his PhD thesis (Theorem 3.5) and also by Jackson and Goulden [24, Lemma 4.2.5]. In the case of the homogeneous complete symmetric function $h_{n}$, it is easy to see that $\left[x_{1} x_{2} \ldots x_{n}\right] h_{k}=\mathbb{1}_{n=k}$. One would like to apply this to the Jacobi-Trudi identity, which is an expression for a Schur function in terms the homogeneous complete symmetric functions:

$$
s_{\lambda}=\operatorname{det}\left(h_{\lambda_{i}+j-i}\right)_{i, j=1}^{\ell(\lambda)} .
$$

The truth is slightly more complicated. Indeed Gordon and Houten did much of the heavy lifting for this problem and had expressed the number of semi-standard tableaux as a determinant of homogenous complete symmetric functions. Gessel extracted the generating function for standard Young tableaux, and derived the formulas in Equation (6).

1.4. The Robinson-Schensted correspondence. The following identity is incredibly evocative to combinatorialists:

$$
\sum_{\lambda \vdash n}\left(f^{\lambda}\right)^{2}=n !
$$

The bijective correspondence between pairs of standard Young tableaux of the same shape and permutations was described by Robinson in the 1930s and also by Schensted in the 1960s. Below we describe the Schensted algorithm which builds a pair of tableaux by parsing the permutation and incrementally building two tableaux. We refer readers to Sagan's book 34 for additional details.

An algorithmic description of the bijection begins with a permutation $\sigma \in \mathbb{S}_{n}$ and a pair of empty tableaux, denoted $\left(P_{0}, Q_{0}\right)$. For $i$ from 1 to $n$ we create $P_{i}$ by adding a box with entry $\sigma(i)$ to $P_{i-1}$ via a special insertion algorithm. For each $i$ we add precisely one box. The position is noted and a box with entry $i$ in the same location is added to $Q_{i-1}$. The sequence of $Q$ tableaux record the history of the box additions. The pair $\left(P_{n}, Q_{n}\right)$ is returned.

The row insertion takes as input a possibly incomplete standard Young tableau and adds an integer $m$ not already in the tableau. The process acts incrementally along each row. If $m$ is bigger than all of the elements in the row under consideration, it is placed at the end of it. If not, it finds its natural place and bumps the larger value. The bumped value is inserted in the tableau formed by the lower rows by the same process.

Example 1. Let $\sigma$ be the involution $\sigma=72961041835$. If the Schensted algorithm is applied, the penultimate step returns the following two tableaux:

$$
P_{9}=\begin{array}{|l|l|l}
\hline 1 & 3 & 8 \\
\hline 2 & 4 & 10 \\
\hline 6 & 9
\end{array} \quad Q_{9}=\begin{array}{|l|l|l|}
1 & 3 & 5 \\
\hline 7 & 4 & 8 \\
\hline 6 & 9 & \\
\hline 7 & &
\end{array} .
$$

The final step is to insert 5 into $P_{9}$. The 5 fits between the 3 and 8 in the first row, hence it bumps 8 to the next row which then bumps 10 which settles at the end of row three. The position of the new square is $(3,3)$, and the algorithm finishes by adding this square to $Q_{9}$, with an entry value of 10 . 
The end result is

$$
P_{10}=Q_{10}=\begin{array}{|l|l|l|}
\hline 1 & 3 & 5 \\
\hline 2 & 4 & 8 \\
\hline 6 & 9 & 10 \\
\hline 7 &
\end{array}
$$

The involution has two fixed points (2 and 8$)$ and the resulting tableaux each have two columns of odd length (specifically, length 3).

The example illustrates some properties which are true in general. The length of the longest increasing subsequence of $\sigma$ is equal to the length of the first row of the tableaux. If $\sigma$ is an involution, then the number of fixed points of $\sigma$ equals the number of columns of odd length in $\lambda$. That standard Young tableaux of size $n$ are equinumerous with involutions was known to Frobenius and Schur.

Viennot 39 described a very beautiful geometric construction using shadow lines to give a more intuitive illustration of this slightly mechanical bijection. It should be better incorporated into the bijections we encounter in later sections. A second geometric construction is given by Fomin using growth diagrams [16]. The construction can be mined for additional information.

1.5. Plan of the article. The first formulas found $Y_{k}(t)$ were recognized to resemble generating functions for walks in Weyl chambers deduced by Grabiner and Magyar [27, Section 6.2]. We examine the relevant developments made in the early 1990s [40, 41, 17, 43, 42] in Section 2 ,

Bijective proofs of some of these connections are more recent [15, 9, 28, although the work of GouyouBeauchamps dates back to the late 1980s. All of these authors' proofs pass through secondary objects, such as coloured Motzkin paths, or matchings. We consider these in Section 2.6 .

One of the most common strategies for enumerating lattice walks restricted to cones with symmetry in the set of allowable steps involves a sub-series extraction from a rational function. Remarkably, many combinatorial classes with transcendental D-finite generating functions share this property. It is an open problem to answer under which conditions this might be universally true. In part, it is a useful formulation since in ideal cases we can answer questions about the order and complexity of the recurrences, and also (re-) derive asymptotic formulas. We describe such extractions, and the resulting tableaux generating functions in Section 3 ,

From the Robinson-Schensted correspondence we see that the combinatorial cousin to the standard Young tableau of bounded height is the permutation with bounded longest increasing subsequence. Many of the techniques described here can also enumerate these classes. For example, Gessel determined expressions for the generating functions, and they also have lattice walk interpretations. We summarize results in Section 4

We conclude with some natural generalizations and open problems.

\section{Lattice Walk Models}

There are no fewer than five different lattice walk classes that are equinumerous with standard Young tableaux of bounded height. Most of them are defined using $d$-dimensional Weyl chamber: $2^{2}$ of type $C$, denoted by $W_{C}(d)$

$$
W_{C}(d) \equiv\left\{\left(x_{1}, x_{2}, \ldots, x_{d}\right): x_{1} \geq x_{2} \geq \cdots \geq x_{d} \geq 0\right\} .
$$

Let $\left\{e_{1}, \ldots, e_{d}\right\}$ denote the standard basis of $\mathbb{R}^{d}$. A lattice walk model is defined by a set of allowable steps and a region which confines each walk. A walk is a sequence of steps. For example, the set

$$
\mathcal{S}=\left\{ \pm e_{i}: 1 \leq i \leq d\right\}
$$

defines the $d$ dimensional simple step set. This is considered in several different bounding cones.

Gessel and Zeilberger [19] considered general walks in Weyl chambers, and demonstrated an enumeration strategy for models where the stepset possesses a certain kind of symmetry, and avoid jumping over boundaries. Such walks have come be called walks reflectable. The generating function for reflectable walk models with specified starting and endpoints (excursions) are written as a coefficient extraction from a signed sum of unrestricted walks. This idea, which appears frequently in lattice walk enumeration, is a version of the reflection principle. The expressions were made explicit for $W_{C}(d)$ by Grabiner and Magyar for the simple step set, which happens to be reflectable.

\footnotetext{
${ }^{2}$ For convenience we define the chambers using non-strict inequalities, our bijective statements can equivalently be given under strict inequalities, upon applying the coordinate shift $\widetilde{x}_{i}=x_{i}+d+1-i$.
} 


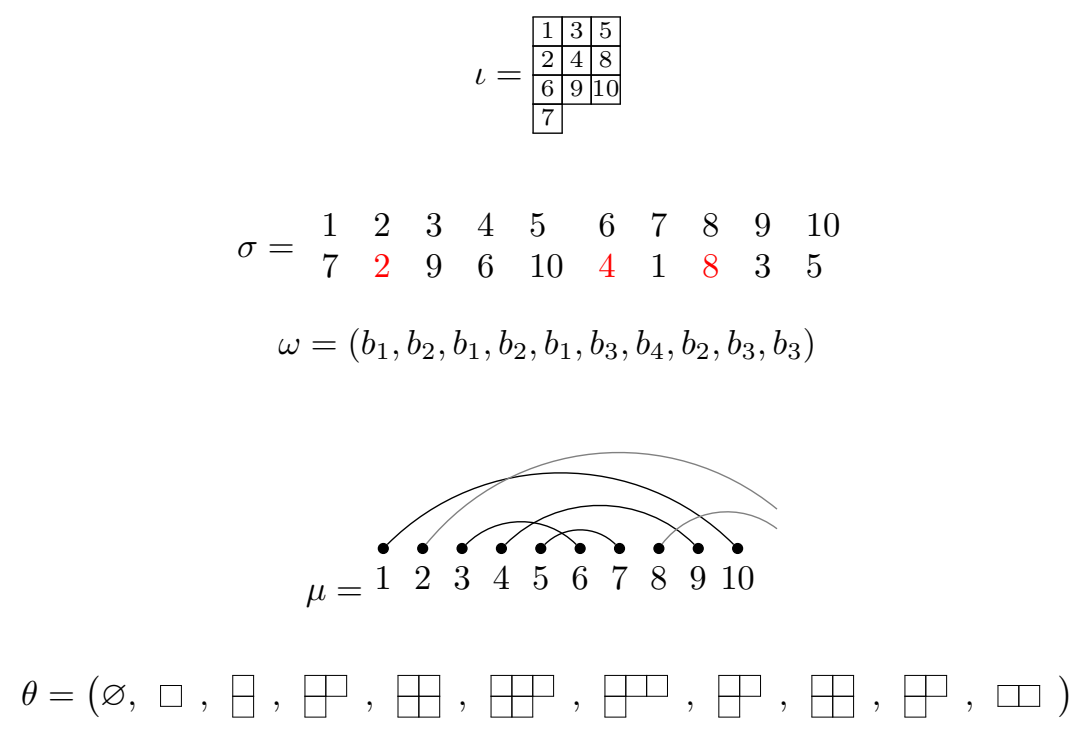

Figure 2. Representatives of classes of objects in bijection with standard Young tableaux of bounded height, in particular their image of $\iota$, a standard Young tableaux of height 3 with two odd columns; $\sigma$ is an involution with maximal increasing sequence of length $3 ; \omega$ is a ballot walk; $\mu$ is an open arc diagram for a partial matching; $\theta$ is an oscillating tableaux ending on a row shape.

Theorem 1 (Grabiner and Magyar 1993 [27]). For fixed $\lambda, \mu \in W_{C}(d)$, the exponential generating function $O_{\lambda, \mu}(t)$ of the simple walks in $W_{C}(d)$ from $\lambda$ to $\mu$, counted by their lengths, satisfies

$$
O_{\lambda, \mu}(t)=\operatorname{det}\left(b_{\mu_{i}-\lambda_{j}}-b_{\mu_{i}+\lambda_{j}}\right)_{1 \leq i, j \leq d} .
$$

This is immediately reminiscent of the generating function formulas in Equation (6). Several authors have made the connection, in particular, Gessel, Weinstein and Wilf 17, Zeilberger [43, Xin [42, Eu et al. [14, 15, Burrill et al. [9] and Courtiel et al. [12. In almost every case there is a natural parameter which is equidistributed with number of odd columns in the tableaux. We describe these classes next.

2.1. Ballot walks. We can build an integer sequence from the entries of a standard Young tableaux. We define the associated lattice word $w=\left(w_{1}, w_{2}, \ldots, w_{n}\right)$ by setting $w_{i}=j$ if the entry $i$ is in the $j$-th row. This word has the property that for any prefix the number of occurrences of $\ell$ is greater than or equal to the number of occurrences of $\ell+1$ since the columns of the associated tableau are strictly decreasing. These are also known as generalized Ballot sequences.

We associate a step naturally to each letter:

$$
w_{1} \rightarrow e_{1}, \quad w_{i} \rightarrow e_{i}-e_{i-1} \quad \text { for }(2 \leq i \leq k-1), \quad w_{k} \rightarrow-e_{k-1} .
$$

Restricting walks to the first orthant is equivalent to the ballot condition. That is, the ballot word of a tableaux of height at most $k$ gives a natural encoding as a lattice walk in the cone $\mathbb{R}_{\geq 0}^{k-1}$ with steps from the following stepset:

$$
\mathcal{B} \equiv\left\{e_{1},-e_{k-1}\right\} \cup\left\{e_{i}-e_{i-1}: 2 \leq i \leq k-1\right\}
$$

Figure 2 has an example.

2.2. Lazy walks. To define this step set, we set $\overline{0}$ to be the zero step (whence the label "lazy"). Zeilberger [43] noted, (although, he attributes the proof to Xin without citation) that the number of excursions in $W_{C}(k)$ starting and ending at the origin using the stepset $\mathcal{L}$, given by

$$
\mathcal{L} \equiv\left\{e_{i},-e_{i}: 1 \leq i \leq k\right\} \cup\{\overline{0}\}
$$




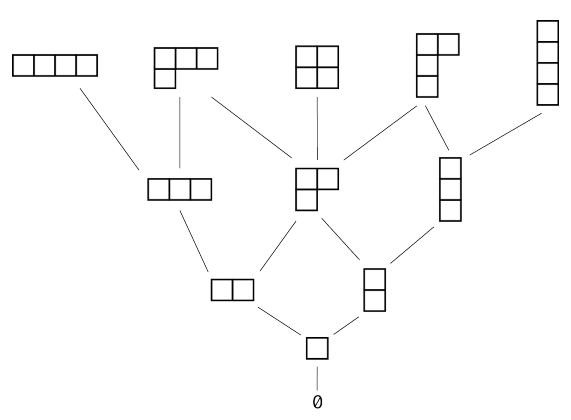

Figure 3. The first few levels of Young's lattice of Ferrers diagrams

is $y_{2 k+1}(n)$. A small computation suggests that the distribution of the zero steps matches the distribution of odd columns in the Young tableaux. He remarks that it would be interesting to find a (bijective) proof of this result.

2.3. Generalized Motzkin Paths. In Zeilberger's lazy walks, the $k=1$ case encodes the classic Motzkin walks, consistent with the longstanding observation that $y_{3}(n)$ is the number of Motzkin words. One could view the higher dimension lazy walks as a generalization of Motzkin words, and this notion was first formalized by $\mathrm{Eu} 14$, and subsequently by $\mathrm{Eu}, \mathrm{Fu}, \mathrm{Hou}$, and $\mathrm{Hsu}$ 15. They add a counter component, and describe an explicit bijection between the Motzkin paths of length $n$ and the standard Young tableaux of size $n$ with at most three rows.

To prove this, they considered the lazy lattice walks, and then algorithmically mapped the steps in. The odd and even cases reconcile as follows.

Theorem 2 (Eu, Fu, Hou, and Hsu 2013 [15, Theorem 1.1]). Consider the lattice model defined by the step set

$$
\mathcal{M} \equiv\left\{e_{1}\right\} \cup\left\{e_{1}+e_{2}, e_{1}-e_{2}\right\} \cup\left\{e_{1}-e_{i}+e_{i+1}, e_{1}+e_{i}-e_{i+1}: 2 \leq i \leq k\right\}
$$

confined to $\mathbb{Z}_{\geq 0}^{k+1}$. The number of walks of length $n$ from the origin to the point $(n, 0, \ldots, 0)$ staying within the nonnegative octant equals the number of $n$-cell SYTs with at most $2 k+1$ rows.

If, additionally, the $e_{1}$ steps are confined to the hyperplane spanned by the vectors $\left\{e_{1}, \ldots, e_{k}\right\}$, then the number of paths equals the number of $n$-cell standard Young tableaux with at most $2 k$ rows.

The number of $e_{1}$ steps is equidistributed with the odd column statistic of standard Young tableaux.

2.4. Oscillating tableaux and arc diagrams. The set of Ferrers diagrams ordered by diagram inclusion ${ }^{3}$ is called Young's lattice. Figure 3 depicts the first few levels of its Hasse diagram. We consider a sequence of Ferrers diagrams as a walk on this lattice. We consider three variants defined by restrictions on moving up or down in the lattice (or not at all). The length of a sequence is the number of elements, minus one. (It is the number of steps in the corresponding walk.)

An oscillating tableau is simply a sequence of Ferrers diagrams such that at every stage a box is either added or deleted. They were popularized by their use in interpretations for representations of the symplectic group [37. If no diagram in the sequence is of height $k+1$, we say that the tableau has its height bounded by $k$. We recall two related families, namely vacillating tableaux, and hesitating tableaux. The vacillating tableaux are even length sequences of Ferrers diagrams, written $\left(\lambda^{(0)}, \ldots, \lambda^{(2 n)}\right)$ where consecutive elements in the sequence are either the same or differ by one square, under the restriction that $\lambda^{(2 i)} \geq \lambda^{(2 i+1)}$ and $\lambda^{(2 i+1)} \leq \lambda^{(2 i+2)}$. The hesitating tableaux are even length sequences of Ferrers diagrams, written $\left(\lambda^{(0)}, \ldots, \lambda^{(2 n)}\right)$ where consecutive differences of elements in the sequence are either the same or differ by one square, under the following restrictions:

- if $\lambda^{(2 i)}=\lambda^{(2 i+1)}$, then $\lambda^{(2 i+1)}<\lambda^{(2 i+2)}$ (do nothing; add a box)

- if $\lambda^{(2 i)}>\lambda^{(2 i+1)}$, then $\lambda^{(2 i+1)}=\lambda^{(2 i+2)}$ (remove a box; do nothing)

- if $\lambda^{(2 i)}<\lambda^{(2 i+1)}$, then $\lambda^{(2 i+1)}>\lambda^{(2 i+2)}$ (add a box; remove a box).

\footnotetext{
${ }^{3}$ Recall $\lambda \leq \mu$ means that $\lambda_{i} \leq \mu_{i}$ for all $i$
} 


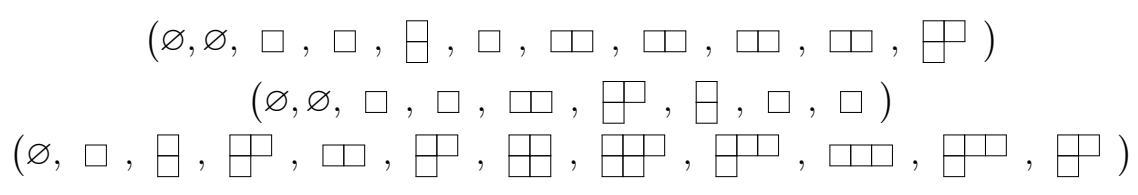

FiguRE 4. From top to bottom: a vacillating tableau of length 10; a hesitating tableau of length 8 ; an oscillating tableau of length 11. In each case, the height is bounded by 2 . From [9]

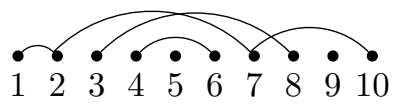

Figure 5. An arc diagram representation of the set partition $\pi=$ $\{1,2,7,10\},\{3,8\},\{4,6\},\{5\},\{9\}$. It is both 3 -noncrossing and 3-nonnesting. However, the subdiagram induced by $\{2,3,7,8,10\}$ is an enhanced 3-crossing. Similarly, the subdiagram induced by $\{3,4,5,6,8\}$ is an enhanced 3 -nesting.

Figure 4 shows examples from each of these classes.

Chen, Deng, Du, Stanley and Yan [1] described non-trivial bijections between sequences of Ferrers diagrams and several combinatorial families encoded in arc diagrams. Arc diagrams are labelled graphs under some degree and embedding restraints. They can be used to represent a variety of combinatorial classes, such as matchings and partitions. Figure 5 illustrates how to encode a set partition as an arc diagram.

They generalize the Schensted algorithm, in some sense, by describing how to parse arc diagrams, with each step defining a tableau insertion or deletion. The result is bijection $\phi$ from partitions to vacillating tableau. It is robust, and can be adapted to other arc diagram classes. A key feature is sub-pattern avoidance properties in the arc diagrams are mapped to height restrictions on the tableaux. This echoes a key feature of the Schensted algorithm.

An arc diagram is noncrossing if no two arcs intersect. Noncrossing set partitions are counted by Catalan numbers. In addition to appearing in combinatorics, these diagrams arise in algebra, physics, and free probability. The notion of a crossing is generalized to a $k$-crossing, which denotes a set of $k$ arcs that each mutually cross. Similarly, a $k$-nesting refers to $k$ arcs which mutually nest into a rainbow figure. More formally, let us consider a set of $k$ distinct arcs: $\left(i_{1}, j_{1}\right), \ldots,\left(i_{k}, j_{k}\right)$. They form a $k$-crossing if $i_{1}<i_{2}<$ $\cdots<i_{k}<j_{1}<j_{2}<\cdots<j_{k}$, and a $k$-nesting if $i_{1}<i_{2}<\cdots<i_{k}<j_{k}<\cdots<j_{2}<j_{1}$. A slightly relaxed notion is sometimes appropriate. Enhanced $k$-nestings and $k$-crossings permit the middle inequality to be an equality. We are interested in the classes of diagrams which avoid such sub-diagrams, and consider $k$ noncrossing diagrams (they contain no $k$-crossing) and $k$-nonnesting diagrams (which contain no $k$-nesting). Figure 5 gives examples.

Theorem 3.2 of Chen et al. [11] proves that given a partition $\pi$ of $\{1, \ldots n\}$, and the vacillating tableau $\phi(\pi)=\left(\emptyset=\lambda^{(0)}, \lambda^{(1)}, \ldots, \lambda^{(2 n)}=\emptyset\right)$, the size of the largest crossing of $\phi$ is the largest height of any $\lambda_{i}$, and the size of the largest nesting is the maximum number of columns.

Thus, by a simple transposition one deduces the corollary comprised of the surprisingly nontrivial fact that $k$-noncrossing set partitions of $\{1,2, \ldots, n\}$ are in bijection with $k$-nonnesting set partitions of $\{1,2, \ldots, n\}$. We leave it to the reader to see why a simple swap of sub-diagrams is not a bijection.

The bijection $\phi$ supports many generalizations. A diagram is said to be open if, in addition to closed edges, there are also half edges. The notion of crossing extends naturally, given a fixed convention of how the open edges lie. The diagram $\mu$ in Figure 2 demonstrates an incomplete, or open, matching. Remark there are not three edges that mutually cross, and hence this diagram is also 3-noncrossing. An open diagram corresponds to a sequence that does not necessarily end at an empty diagram- rather it ends in a row shape, i.e. a partition with a single part: $(m)$. The bijection $\phi$ is well defined in this case, although Chen et al. did 
not discuss how to interpret this, although Burrill, Melczer and Mishna [10]. Xin [42] consider walks with any endpoint, and found generating functions for palindromic sequences.

In a sequence of Ferrers diagrams of height at most $k$, each diagram can be coded by a $k$-dimensional vector where each successive entry is weakly decreasing. Thus, a sequence is an encoding of a walk in $W_{C}(d)$. Thus we can view the bijection $\phi$ as a nontrivial map between arc diagrams and walks. To end in a row shape is the same as ending on the first axis.

This brings us back to our topic at hand, standard Young tableaux. Which arc diagrams are associated to a standard Young tableau of bounded height? The most plain interpretation is to view a standard Young tableau as a sequence of Ferrers diagrams - it starts at the empty diagram and a single cell is added at each step, with the position indicated by the entry in the tableaux. We could consider the arc diagram image of this sequence, but we can do better.

Recall the Robinson-Schensted correspondence is a bijection between pairs of standard Young tableaux and permutations. By restricting the map to pairs of identical tableaux, it becomes a bijection between standard Young tableaux and involutions. Involutions have a very natural arc diagram representation! In this correspondence a tableau with a fixed number of odd columns is mapped to an involution with precisely that many fixed points. An involution is a partial matching, and they are in bijection with oscillating tableaux.

These pieces lead to the following result, a map between standard Young tableaux and a class of walks that end on a boundary.

Theorem 3 (Krattenthaler 2016 [28, Theorem 4]; Burrill, Courtiel, Fusy, Melczer and Mishna 2016 [9, Theorem 1]; Okada 2016 [30, Theorem 1.2]). For $n, k \geq 1$, there is an explicit bijection between the standard Young tableaux of size $n$ with height at most $k$ and with $m$ odd columns, and the simple walks of length $n$ staying in $W_{C}(k)$, starting from the origin and ending at the point $m e_{1}$.

We have outlined one possible argument, but in fact three very different proofs have been discovered. Above is the argument of Burrill, Courtiel, Fusy, Melczer and Mishna 9]. Krattenthaler 28] determines a different bijection using growth diagrams as an intermediary object. It is direct, and gives several generalizations. It is self contained in that it does not exploit the $\tau$ map. Okada [30] uses a representation theoretic argument starting from Pieri rules. He also has generalizations to semi-standard tableaux. Gouyou-Beauchamps proved the $k=4$ case.

One consequence of a lattice walk bijection is a generating function expression. Applying Grabiner and Magyar's formula gives the following

$$
Y_{k}(t)=\sum_{u=0}^{k-1}(-1)^{u} \sum_{\ell=u}^{2 k-1-2 u}\left(b_{\ell}\right) \operatorname{det}\left(b_{i-j}-b_{k d-i-j}\right)_{0 \leq i \leq k-1, i \neq u, 1 \leq j \leq k-1} .
$$

Remarkably, the infinite sum which arises from direct application of Grabiner and Magyar's formula telescopes into a finite sum. We also use the identity $b_{-k}=b_{k}$.

2.5. Excursions in the Weyl chamber of type D. Classically, there is a simple bijection between walks with steps from $\{(1,1),(1,-1)\}$ which, in the first instance start and end on the axis, with no further restriction (let us call them bridges), and in the second instance start at the origin, and never go below the axis and can end at any height (Dyck prefixes). The first class restricts free walks by restricting the possible end points, and the second restricts free walks by restricting the size of the region. We can view this as a tradeoff of restrictions. The simplest proof of this result passes through a third object, marked Dyck paths, which are objects in the intersection of both classes, but have additional markings on some of the down steps which touch the axis. Consider the following two maps, which both define bijections. A Dyck path with marked down steps is mapped to a Dyck prefix, by flipping all of the marked down steps into upsteps. The flipped marked steps become the last step at that height in the walk, and the Dyck prefix ends at a height equal to the number of marked steps. On the other hand, we could consider the entire segment of the walk ending at a marked down step, starting at the nearest previous up step which touched the axis. We can flip this entire segment across the axis. We do this for every marked step to build a bridge. The marked intermediary facilitates a straightforward proof, but how to mark in higher dimensions? 
The Weyl chamber of type $D$ is the following region:

$$
W_{D}(k) \equiv\left\{\left(x_{1}, x_{2}, \ldots, x_{k}\right) \quad: \quad x_{1} \geq x_{2} \geq \cdots \geq x_{k-1} \geq\left|x_{k}\right|\right\} .
$$

An axis-walk is any walk starting at the origin and ending on the $x_{1}$-axis.

Theorem 4 (Courtiel, Fusy, Lepoutre and Mishna 2018 [12, Theorem 20]). For $k \geq 1$ and $n \geq 0$, there is an explicit bijection between simple axis-walks of length $n$ staying in $W_{C}(k)$ and simple excursions of length $n$ staying in $W_{D}(k)$, starting from $\left(\frac{1}{2}, \ldots, \frac{1}{2}, \frac{1}{2}\right)$, and ending at $\left(\frac{1}{2}, \ldots, \frac{1}{2}, \frac{(-1)^{n}}{2}\right)$. The ending $x_{1}$-coordinate of a walk from $W_{C}(k)$ corresponds to the number of steps that change the sign of $x_{k}$ in its bijective image.

The proof of this result is a generalization of the marked Dyck path example. The analog to the Dyck prefix is the axis walk in the orthant space $W_{C}(k)$, and the analog to the bridge is the excursion in the larger space $W_{D}(k)$. The intermediary class, marked excursions in $W_{C}(k)$, are defined by a less straightforward process. For the first bijection, Courtiel et al. map the axis walks to open arc diagrams. The open arcs are removed, but their position is marked. The inverse bijection is applied and a marked excursion results. The second bijection is slightly more complicated, and the reader is referred to the article for details.

A corollary of this result is another lattice model in bijection with standard Young tableaux of bounded height.

Corollary 5 (Courtiel, Fusy, Lepoutre, Mishna 2018 [12, Corollary 21]). For $n, k \geq 1$, there is an explicit bijection between the standard Young tableaux of size $n$ with height at most $k$, and the simple walks of length $n$ staying in $W_{D}(k)$, starting from $\left(\frac{1}{2}, \ldots, \frac{1}{2}, \frac{1}{2}\right)$, and ending at $\left(\frac{1}{2}, \ldots, \frac{1}{2}, \frac{(-1)^{n}}{2}\right)$. The number of odd columns corresponds to the number of steps that change the sign of $x_{k}$.

It seems very likely that an explicit bijection could be found between these, and the lazy walks. This would answer the question of Zeilberger, and likely reveal a model for the odd cases.

There is also potential for another generating function formula by applying the results of Grabiner and Magyar.

2.6. A collection of bijections. We summarize some combinatorial classes in bijection with standard Young tableaux of bounded height. Figure 2 illustrates some of these classes.

Theorem 6. The set of standard Young tableaux of size $n$ with height bounded by $k$ and $m$ odd columns is in bijection with each of the following sets:

(1) The set of involutions of size $n$ with $m$ fixed points and no decreasing subsequence of length $k+1$;

(2) The set of oscillating tableaux of size $n$ with height bounded by $k$, which start at the empty partition and end in a row shape $\lambda=(m)$;

(3) (if $k$ is even) The set of open matching diagrams of length $n$, with $m$ open arcs and with no $(k / 2+1)$ crossing;

Gil, McNamara, Tirrell and Weiner [20, Theorem 1.1] proved the equivalence between Class (3) in the theorem, and a set of a Dyck paths where each groups of ascent steps are decorated by a connected matching of a related size. A main feature of their construction is the following identity. Suppose $P_{k}(t)$ denotes the generating function for the number of $k$-noncrossing perfect matchings on [2n], then

$$
Y_{2 k-1}(t)=\frac{1+P_{k}\left(t^{2}(1-t)^{-2}\right)}{1-t} .
$$

\section{Generating function expressions}

The generating functions of lattice walks in cones with symmetries in the stepset can often be expressed as a subseries extraction of a Laurent expansion of a rational function. This generalizes the result on reflectable walks of Gessel and Zeilberger, and is at the heart of the orbit sum method of Bousquet-Mélou and Mishna 7. which handles a wide class of lattice walks restricted to the quarter plane. In their work, they start with a natural combinatorial recurrence on lattice walks, which translates to a functional equation satisfied by the generating function. For some models it is sufficient to take a weighted sum (the namesake orbit sum) of the equations, and isolate the target generating function with a sub-series extraction. 
In this context, the extraction operates on iterated Laurent series. For a function $F\left(x_{1}, \ldots, x_{d} ; t\right) \in$ $\mathbb{C}\left(x_{1}, \ldots, x_{d}\right)(t)$ which is analytic at the origin, we denote by $\left[x_{1}^{k_{1}} x_{2}^{k_{2}} \ldots x_{d}^{k_{d}} t^{n}\right] F\left(x_{1}, \ldots, x_{d} ; t\right)$ to be the coefficient of the term $x_{1}^{k_{1}} x_{2}^{k_{2}} \ldots x_{d}^{k_{d}} t^{n}$ in the Laurent expansion. In this context we view the objects to be series in $t$ variable. We recall the terminology that several authors use for the special case of the constant term with respect to a set of variables: $\mathrm{CT}_{x_{1} \ldots x_{d}} F\left(x_{1}, \ldots, x_{d} ; t\right)$ is the coefficient of the term $x_{1}^{0} \ldots x_{d}^{0}$ in the Laurent series expansion of $F$ at the origin. It is a series in the remaining variables. It can be obtained by incrementally determining the constant term with respect to a single variable. Here we shall take the order of the variables as they are listed, for example. Such a coefficient extraction can be written as a Cauchy integral.

A related operator is the diagonal. The (central) diagonal $\Delta F\left(x_{0}, x_{1}, \ldots, x_{d}\right)$ of a formal power series is the univariate subseries defined

$$
\Delta F\left(x_{0}, \ldots, x_{d}\right)=\Delta \sum_{i_{0}, \ldots, i_{d}} f\left(i_{0}, \ldots, i_{d}\right) x_{0}^{i_{0}} \ldots z_{d}^{i_{d}} \equiv \sum_{n} f(n, \ldots, n) t^{n} .
$$

Lipshitz [29] proved that the diagonal of a D-finite function $]^{4}$ is also D-finite. Since rational functions are D-finite, diagonals of rational functions are also D-finite. The expressions we consider here are all diagonals of rational functions, and hence are all D-finite by construction. This provides an alternate proof of the D-finiteness of $Y_{k}(t)$.

Bousquet-Mélou [6] used an orbit sum to directly derive generating function expressions for standard Young tableaux of bounded height. Her generating functions also mark the number of odd columns, and other parameters are also readily accessible. In that same paper, she determines a nice functional equation proof of the hook length formula. The starting point is a simple recursive construction for standard Young tableaux: a tableau of size $n+1$ is obtained from a tableau of size $n$ by adding a cell to the $j$-th row, unless the $j$-th row is already the same length as the $j-1$ row. This translates into a very straightforward functional equation for the generating function

$$
F(u) \equiv \sum_{\substack{\lambda \vdash n \\ \ell(\lambda) \leq k}} f^{\lambda} u_{1}^{\lambda_{1}} u_{2}^{\lambda_{2}} \cdots .
$$

The equation is defined in term of $F_{j}(u)$, the generating function for those standard Young tableaux such that $\lambda_{j-1}=\lambda_{j}$ :

$$
F(u)=1+u_{1} F(u)+\sum_{j=2}^{k} u_{j}\left(F(u)-F_{j}(u)\right) .
$$

She applies a kernel method argument to this functional equation to recover MacMahon's formula for $f^{\lambda}$.

She has generating function expressions in her Propositions 9 and 10. It is her Proposition 11 that accounts for the additional parameter of interest. We note that she works with the ordinary generating function,

$$
\widetilde{Y}_{k}(u ; t) \equiv \sum_{n} \sum_{\substack{\lambda \vdash n \\ \ell(\lambda) \leq k}} f^{\lambda} u^{\# \text { odd columns in } \lambda} t^{n} .
$$

Theorem 7 (Bousquet-Mélou 2011 [6]). For $k=2 \ell$, the ordinary generating function of standard Young tableaux with height bounded by $k$ where $t$ marks the length and $x_{1}$ marks the number of odd columns is

$$
\widetilde{Y}_{k}\left(x_{1} ; t\right)=\mathrm{CT}_{x_{2}, \ldots, x_{k}} \frac{-\operatorname{det}\left(x_{j}^{-i}-x_{j}^{i}\right)_{1 \leq i, j \leq \ell}}{1-t\left(x_{1}+x_{1}^{-1}+\cdots+x_{\ell}+x_{\ell}^{-1}\right)} \frac{1}{x_{2} x_{3}^{2} \ldots x_{\ell}^{\ell-1}} .
$$

The orbit sum strategy can be used to determine an expression for the ordinary generating function for simple walks in $\mathcal{W}_{C}(k)$ ending on the axis. This gives the following result.

\footnotetext{
${ }^{4} \mathrm{~A}$ (multivariate) function is D-finite if the set of all its partial derivatives spans a vector space of finite dimension.
} 
Theorem 8 (Burrill, Melczer and Mishna 2015 [10]). The ordinary generating function, $\widetilde{Y}_{k}(t)$ for Young tableaux of height bounded by $k$ satisfies the formula formula

$$
\begin{aligned}
& t^{2 k-1} \widetilde{Y}_{2 k}(t)= \\
& -\Delta\left[\frac{z_{0}^{2 k-1}\left(z_{3} z_{4}^{2} \cdots z_{k}^{k-2}\right)\left(z_{1}+1\right) \prod_{1 \leq j<i \leq k}\left(z_{i}-z_{j}\right)\left(z_{i} z_{j}-1\right) \cdot \prod_{2 \leq i \leq k}\left(z_{i}^{2}-1\right)}{1-z_{0}\left(z_{1} \cdots z_{k}\right)\left(z_{1}+z_{1}^{-1}+\cdots z_{k}+z_{k}^{-1}\right)}\right] .
\end{aligned}
$$

These formulas are not necessarily easier to compute or interpret than the others, but they do have a few implications. They do provide a proof that the generating function is D-finite. Furthermore, we see a second proof that $y_{k}(n)$ can be expressed as binomial sums, and potentially a different route to obtain such expressions, using the work of Bostan, Lairez and Salvy [5. They may be suitable for analysis by the methods of Pemantle and Wilson [31, which determine asymptotic formulas for coefficients of functions which are expressed as diagonals of rational functions.

Since it is the generating function for Motzkin numbers, $\widetilde{Y}_{3}(t)$ is algebraic. Because the asymptotics of the coefficients are incompatible with algebraicity, $\widetilde{Y}_{4}(t)$ is not. For which $k$ is the ordinary generating function $\widetilde{Y}_{k}(t)$ algebraic?

3.1. Differential equations. Once we know that a generating function is D-finite, it is natural to ask about differential equations that it satisfies. Bergeron, Favreau and Krob 2 generated many conjectures about the order of the differential equation satisfied by $Y_{k}(t)$ from computer experiments, and some analysis of its expression as a determinant of a matrix of modified Bessel functions.

Proposition 1 in [3] states the dimension of the vector space over the field $\mathbb{C}(t)$ of rational functions in $t$ spanned by $Y_{k}(t)$ and all its derivative is bounded by $\left\lfloor\frac{k}{2}\right\rfloor$.

Conjecture 9 (Bergeron, Favreau, Krob 1995 [2]). For each $k$, there are polynomials $p_{m}(t)$ of degree at most $\left\lfloor\frac{k}{2}\right\rfloor$ such that $Y_{k}(t)$ is a solution of a linear differential equation order at most $\left\lfloor\frac{k}{2}\right\rfloor+1$ with coefficients $p_{m}(t)$.

They have verified this conjecture for $k \leq 11$.

We can use recent advances in symbolic computation and the diagonal expression in Theorem 8 to bound the order and degree of the differential equations satisfied by the ordinary generating function $\widetilde{Y}_{k}(t)$. In particular, the following theorem of Bostan, Lairez, and Salvy [4] can be explicitly applied.

Theorem 10 (Bostan, Lairez, and Salvy [4]). Let $R\left(z_{1}, \ldots, z_{d}, t\right)=A(\mathbf{z}, t) / B(\mathbf{z}, t) \in \mathbb{Q}(t)\left(z_{1}, \ldots, z_{d}\right)$, be $a$ rational function with multidegree bounds

$$
n_{\mathbf{z}}:=\max \left(\operatorname{deg}_{\mathbf{z}} B, \operatorname{deg}_{\mathbf{z}} A+d+1\right) \quad n_{t}:=\max \left(\operatorname{deg}_{t} A, \operatorname{deg}_{t} B\right) .
$$

Then there exists an annihilating differential equation $\mathcal{L}$ for the integral

$$
P(t):=\oint_{\gamma} R(\mathbf{z}, t) d \mathbf{z},
$$

where $\gamma$ is any $n$-cycle in $\mathbb{C}^{n}$ on which $R$ is continuous when $t$ ranges over some connected open set $U \subset \mathbb{C}$ (note that $\mathcal{L}$ is independent of $\gamma$ ). Furthermore the order of $\mathcal{L}$ is at most $n_{\mathbf{z}}^{d}$ and the degree of $\mathcal{L}$ is at most $\left(\frac{5}{8} n_{\mathbf{z}}^{3 d}+n_{\mathbf{z}}^{d}\right) e^{d} n_{t}$.

We write a diagonal as an integral:

$$
\Delta F(\mathbf{z}, t)=\left(\frac{1}{2 \pi i}\right)^{d} \int_{\gamma} \frac{F\left(z_{1}, z_{2} / z_{1}, z_{3} / z_{2}, \ldots, z_{d} / z_{d-1}, t / z_{d}\right)}{z_{1} z_{2} \cdots z_{d}} d \mathbf{z}
$$

by the multivariate Cauchy residue theorem, for an appropriate $n$-cycle $\gamma$ around the origin.

In the case of $\widetilde{Y}_{k}(t)$, we compute an upper bound of $2 d^{2}-3 d+1$ on the total degree of the denominator in the $\mathbf{z}$ variables and a bound of $2 d^{2}-4 d-2$ on the degree of the numerator and implies the following result, computed by Melczer.

Proposition 11. The generating function for the number of standard Young tableaux of height at most $k$ satisfies a linear differential equation of order at most $\left(2 k^{2}-3 k+1\right)^{k}$ and degree at most

$$
\left(\frac{5}{8}\left(2 d^{2}-3 d+1\right)^{3 d}+\left(2 d^{2}-3 d+1\right)^{d}\right) e^{d} .
$$


3.2. Asymptotics. Regev [32 determined asymptotic expansions for the number of Young tableaux of bounded height. He explicitly deduced asymptotics for the $k=3$ case as a particular extraction:

$$
(n+1) y_{3}(n)=\left[x^{-1}\right](x+1 / x+1)^{n+1} \sim \sqrt{\frac{3}{8} \pi} \cdot \frac{1}{\sqrt{n}} 3^{n} .
$$

By a slightly more general argument, he showed

$$
y_{2 k}(n) \sim_{n \rightarrow \infty}(2 / \pi)^{k / 2}(2 k)^{n}(k / n)^{k(k-1 / 2)} \prod_{i=0}^{k-1}(2 i) !
$$

The asymptotics of lattice walks in cones have been well studied. The tour de force of Denisov and Wachtel [13] describes a collection of very comprehensive results. The formulas given in [13, Theorem 6] should be applied here, for example to the lazy walks and to the excursions in $W_{D}(k)$.

Grabiner [26] used his formulas for walks in Weyl chambers to find the asymptotics of the probability that a randomly chosen standard Young tableau of size $n$ with at most $t$ rows contains a given subtableau. This is equivalent to counting walks that have visited a particular point- there might be similar results to be extracted by considering other lattice models.

The asymptotic formula that have been described so far are valid for fixed $k$, as $n$ tends to infinity. It is open to develop formulas when $k$ is a function of $n$.

\section{RESTRICTING INCREASING SUBSEQUENCES IN PERMUtATIONS}

Motivated by algebraic interpretations, Regev [32] considered the quantity

$$
y_{k}^{(\beta)}(n) \equiv \sum_{\lambda \in \mathcal{P}_{k}}\left(f^{\lambda}\right)^{\beta}
$$

What can be said of the generating functions

$$
\sum y_{k}^{(\beta)}(n) t^{n} \quad \sum y_{k}^{\beta}(n) t^{n} u^{\beta} ?
$$

When $\beta=2$, this counts permutations with restricted longest increasing subsequence, and it is very well studied, with many relevant connections to lattice walks [41, 40. Define $u_{k}(n)$ to be the number of pairs of Young tableaux of the same shape with at most $k$ rows. By the Robinson-Schensted correspondence, this is the number of permutations in $\mathfrak{S}_{n}$ with no $(k+1)$-increasing subsequence. For every $k \geq 1$, Gessel [18] proved the formula

$$
\sum_{n \geq 0} \frac{u_{k}(n)}{n !^{2} t^{2 n}}=\operatorname{det}\left(b_{i-j}\right)_{1 \leq i, j \leq k},
$$

where the $b_{j}$ are the Bessel function evaluations defined earlier.

A combinatorial proof of this expression has been given by Gessel et al. 17] via simple walks ending at so-called Toeplitz points, and Xin [42] did a combinatorial derivation based on arc diagrams, and yet another constant term extraction. The permutations satisfy a nice combinatorial recursion, and, in a manner similar to her solution for involutions, Bousquet-Mélou [6] determines functional equations that can be resolved using a kernel method approach.

She determined [6, Proposition 13] the following expression for the ordinary generating function of permutations avoiding the pattern $12 \ldots m(m+1)$ :

$$
U_{k}(t) \equiv \sum_{n \geq 0} u_{k}(n) t^{2 n}=\mathrm{CT}_{x_{1}, \ldots, x_{m}} \frac{\operatorname{det}\left(\left(x_{j}-x_{j-1}\right)^{i-j}\right)_{1 \leq i, j \leq m}}{1-t\left(\sum \frac{1}{x_{j}-x_{j-1}}\right)} \cdot \sum_{i=0}^{m} \prod_{j=1}^{i} \frac{x_{j}}{1-x_{j}} .
$$

Bergeron and Gascon found the differential equations satisfied by the exponential generating functions for $k<11$. It remains open to answer if $\left(y_{k}^{(\beta)}(n)\right)$ is the counting sequence for any easily characterizable combinatorial family, perhaps as a restricted or decorated family of permutations. The argument of Gessel [18] applies to prove that the sequence is P-recursive for positive integer $\beta$, and probably also a diagonal of a rational function. Can we usefully bound the annihilating differential operators or determine asymptotic formulas for arbitrary $\beta$ ? 
Wilf [40] deduced $U_{2 k}(t)=Y_{2 k}(t) Y_{2 k}(-t)$. From this, it follows

$$
\left(\begin{array}{c}
2 n \\
n
\end{array}\right) u_{2 k}(n)=\sum_{r}\left(\begin{array}{c}
2 n \\
r
\end{array}\right)(-1)^{r} y_{2 k}(r) y_{2 k}(2 n-r) .
$$

Is there a lattice path interpretation of this identity? Are there identities for other $\beta$ values?

\section{Other DiRECtions}

5.1. Using Kronecker coefficients. The Kronecker product of symmetric functions gives an important connection to representation theory. In particular, the Kronecker product of two symmetric functions in the Schur function basis determines the multiplicities of irreducible characters in this tensor product.

The following Schur function identity for the Kronecker product of two Schur functions (denoted by *) was shown by Brown, van Willigenburg and Zabrocki [8]:

$$
s_{(n, n-1)} * s_{(n, n-1)}=\sum_{\substack{\lambda \vdash 2 n-1 \\ \ell(\lambda) \leq 4}} s_{\lambda} .
$$

Tewari [38, manipulates this formula to deduce a closed form for the number of Young tableaux with height exactly 5 , under the additional constraint of $\lambda_{5}=1$. His Theorem 7.4 is a simple sum of Motzkin numbers (recall $M_{n}=y_{3}(n)$ ) and pairs of Catalan numbers (recall also the formula of $y_{4}$ ):

$$
\begin{aligned}
& \sum_{\substack{\lambda+2 n \\
\ell(\lambda)=5 \\
\lambda_{5}=1}} f^{\lambda}=\left(\frac{n(n+2)}{2 n+1}\right) C_{n} C_{n+1}-C_{n+1}^{2}+M_{2 n}, \\
& \sum_{\substack{\lambda+2 n-1 \\
\ell(\lambda)=5 \\
\lambda_{5}=1}} f^{\lambda}=\left(\frac{(n+1)}{2}\right) C_{n}^{2}-C_{n} C_{n+1}+M_{2 n-1} .
\end{aligned}
$$

It could be straightforward to find a combinatorial interpretation of his formula, which then could be generalized to higher dimensions. It also suggests that perhaps some of the larger summations could be simplified. This could be key to finding a useful expressions for $y_{6}(n)$.

5.2. Other classes in bijection. As Gouyou-Beauchamps noted in [25], the numbers that appear in $y_{4}(n)$ also appear in the enumeration of planar maps and alternating Baxter permutations. Baxter permutations are a class of pattern avoiding permutations that are very combinatorially rich. They also have a bijection to lattice path models, using the machinery of arc diagrams as an intermediary class. It might be possible to directly connect these classes.

5.3. Shadow diagrams. Some of the lattice walk bijections use tableau insertion and deletion in more than one stage. Perhaps there exist more economical, or direct bijections. The shadow diagrams of Viennot may play an important role in such a simplification.

5.4. Random tableaux. There have been several recent works on generating random walks. One application is to convert this to a generator for random tableaux. Which of the above bijections has smallest complexity?

Grabiner [26] was able to use lattice walk results to determine distributions of subtableaux in Young tableaux. It seems that there should be more results along this vein with each of the lattice walk representations.

5.5. Semi-standard Young tableaux. Okada 30 proved some results connecting counting sequences of generalized oscillating tableaux and semi-standard tableaux using techniques from representation theory. In particular, his Theorem 5.3 is a list of results on equinumerous classes of tableaux of bounded height that are well suited for more bijective explanations.

Krattenthaler's results [28] on semi-standard tableaux replace single steps in oscillating tableaux with jumps by horizontal strips. Perhaps they can connect here using lattice walks with longer steps or diagonal steps. In any case, these two works should be connected more explicitly. 


\section{ACKNOWLEDGEMENTS}

The author is grateful to MSRI for travel support to participate in the 2017 AWM session. This expository work was inspired by that meeting. I am grateful for the patience and wisdom of the anonymous referees. The author's research is also partially funded by NSERC Discovery Grant RGPIN-04157.

\section{REFERENCES}

[1] Ron Adin and Yuval Roichman. Standard Young tableaux. In Handbook of enumerative combinatorics, Discrete Math. Appl. (Boca Raton), pages 895-974. CRC Press, Boca Raton, FL, 2015.

[2] François Bergeron, Luc Favreau, and Daniel Krob. Conjectures on the enumeration of tableaux of bounded height. Discrete Mathematics, 139(1-3):463-468, 1995.

[3] François Bergeron and Francis Gascon. Counting Young tableaux of bounded height. Journal of Integer Sequences, 3(2):3, 2000.

[4] Alin Bostan, Pierre Lairez, and Bruno Salvy. Creative telescoping for rational functions using the Griffiths-Dwork method. In ISSAC 2013-Proceedings of the 38th International Symposium on Symbolic and Algebraic Computation, pages 93-100. ACM, New York, 2013.

[5] Alin Bostan, Pierre Lairez, and Bruno Salvy. Multiple binomial sums. J. Symbolic Comput., 80(part 2):351-386, 2017.

[6] Mireille Bousquet-Mélou. Counting permutations with no long monotone subsequence via generating trees and the kernel method. J. Algebraic Combin., 33(4):571-608, 2011.

[7] Mireille Bousquet-Mélou and Marni Mishna. Walks with small steps in the quarter plane. In Algorithmic probability and combinatorics, volume 520 of Contemp. Math., pages 1-39. Amer. Math. Soc., Providence, RI, 2010.

[8] Andrew A. H. Brown, Stephanie van Willigenburg, and Mike Zabrocki. Expressions for Catalan Kronecker products. Pacific J. Math., 248(1):31-48, 2010.

[9] Sophie Burrill, Julien Courtiel, Eric Fusy, Stephen Melczer, and Marni Mishna. Tableau sequences, open diagrams, and Baxter families. European J. Combin., 58:144-165, 2016.

[10] Sophie Burrill, Stephen Melczer, and Marni Mishna. A Baxter class of a different kind, and other bijective results using tableau sequences ending with a row shape. In Proceedings of FPSAC 2015, Discrete Math. Theor. Comput. Sci. Proc., pages 369-380. Assoc. Discrete Math. Theor. Comput. Sci., Nancy, 2015.

[11] William Y. C. Chen, Eva Y. P. Deng, Rosena R. X. Du, Richard P. Stanley, and Catherine H. Yan. Crossings and nestings of matchings and partitions. Trans. Amer. Math. Soc., 359(4):1555-1575, 2007.

[12] Julien Courtiel, Eric Fusy, Mathias Lepoutre, and Marni Mishna. Bijections for Weyl chamber walks ending on an axis, using arc diagrams and Schnyder woods. European Journal of Combinatorics, 69:126-142, March 2018.

[13] Denis Denisov and Vitali Wachtel. Random walks in cones. Ann. Probab., 43(3):992-1044, 2015.

[14] Sen-Peng Eu. Skew-standard tableaux with three rows. Adv. in Appl. Math., 45(4):463-469, 2010.

[15] Sen-Peng Eu, Tung-Shan Fu, Justin T. Hou, and Te-Wei Hsu. Standard Young tableaux and colored Motzkin paths. J. Combin. Theory Ser. A, 120(7):1786-1803, 2013.

[16] Sergey Fomin. Schensted algorithms for dual graded graphs. J. Algebraic Combin., 4(1):5-45, 1995.

[17] Ira Gessel, Jonathan Weinstein, and Herbert S. Wilf. Lattice walks in $\mathbf{Z}^{d}$ and permutations with no long ascending subsequences. Electron. J. Combin., 5:Research Paper 2, 11, 1998.

[18] Ira M. Gessel. Symmetric functions and P-recursiveness. J. Combin. Theory Ser. A, 53(2):257-285, 1990.

[19] Ira M. Gessel and Doron Zeilberger. Random walk in a Weyl chamber. Proc. Amer. Math. Soc., 115(1):27-31, 1992.

[20] Juan B Gil, Peter RW McNamara, Jordan O Tirrell, and Michael D Weiner. From Dyck paths to standard Young tableaux. arXiv preprint arXiv:1708.00513, 2017.

[21] Basil Gordon. Notes on plane partitions. IV. Multirowed partitions with strict decrease along columns. In Combinatorics (Proc. Sympos. Pure Math., Vol. XIX, Univ. California, Los Angeles, Calif., 1968), pages 91-100. Amer. Math. Soc., Providence, R.I., 1971.

[22] Basil Gordon and Lorne Houten. Notes on plane partitions. I, II. J. Combinatorial Theory, 4:72-80; 81-99, 1968.

[23] Basil Gordon and Lorne Houten. Notes on plane partitions. III. Duke Math. J., 36:801-824, 1969.

[24] I. P. Goulden and D. M. and Jackson. Combinatorial enumeration. A Wiley-Interscience Publication. John Wiley \& Sons, Inc., New York, 1983. With a foreword by Gian-Carlo Rota, Wiley-Interscience Series in Discrete Mathematics.

[25] Dominique Gouyou-Beauchamps. Standard Young tableaux of height 4 and 5. European J. Combin., 10(1):69-82, 1989.

[26] David J. Grabiner. Asymptotics for the distributions of subtableaux in Young and up-down tableaux. Electron. J. Combin., 11(2):Research Paper 29, 22, 2004/06.

[27] David J. Grabiner and Peter Magyar. Random walks in Weyl chambers and the decomposition of tensor powers. J. Algebraic Combin., 2(3):239-260, 1993.

[28] C. Krattenthaler. Bijections between oscillating tableaux and (semi)standard tableaux via growth diagrams. J. Combin. Theory Ser. A, 144:277-291, 2016.

[29] L. Lipshitz. The diagonal of a $D$-finite power series is D-finite. J. Algebra, 113(2):373-378, 1988.

[30] Soichi Okada. Pieri rules for classical groups and equinumeration between generalized oscillating tableaux and semistandard tableaux. Electron. J. Combin., 23(4):Paper 4.43, 27, 2016.

[31] Robin Pemantle and Mark C. Wilson. Analytic combinatorics in several variables, volume 140 of Cambridge Studies in Advanced Mathematics. Cambridge University Press, Cambridge, 2013. 
[32] Amitai Regev. Asymptotic values for degrees associated with strips of Young diagrams. Adv. in Math., 41(2):115-136, 1981.

[33] Bruce E. Sagan. The ubiquitous Young tableau. In Invariant theory and tableaux (Minneapolis, MN, 1988), volume 19 of IMA Vol. Math. Appl., pages 262-298. Springer, New York, 1990.

[34] Bruce E. Sagan. The symmetric group, volume 203 of Graduate Texts in Mathematics. Springer-Verlag, New York, second edition, 2001. Representations, combinatorial algorithms, and symmetric functions.

[35] R. P. Stanley. Differentiably finite power series. European J. Combin., 1(2):175-188, 1980.

[36] Richard P. Stanley. Increasing and decreasing subsequences and their variants. In International Congress of Mathematicians. Vol. I, pages 545-579. Eur. Math. Soc., Zürich, 2007.

[37] Sheila Sundaram. The Cauchy identity for $\operatorname{Sp}(2 n)$. J. Combin. Theory Ser. A, 53(2):209-238, 1990.

[38] Vasu V. Tewari. Kronecker coefficients for some near-rectangular partitions. J. Algebra, 429:287-317, 2015.

[39] G. Viennot. Une forme géométrique de la correspondance de robinson-schensted. In D. Foata, editor, Combinatoire et repreésentation du groupe symétrique. Lecture Notes in Mathematics, volume 579, pages 29-58. Springer-Verlag, Berlin, 1978.

[40] Herbert S. Wilf. Ascending subsequences of permutations and the shapes of tableaux. J. Combin. Theory Ser. A, 60(1):155$157,1992$.

[41] Herbert S. Wilf. The computer-aided discovery of a theorem about Young tableaux. J. Symbolic Comput., 20(5-6):731-735, 1995. Symbolic computation in combinatorics $\Delta_{1}$ (Ithaca, NY, 1993).

[42] Guoce Xin. Determinant formulas relating to tableaux of bounded height. Adv. in Appl. Math., 45(2):197-211, 2010.

[43] Doron Zeilberger. The number of ways of walking in $x_{1} \geq \cdots \geq x_{k} \geq 0$ for $n$ days, starting and ending at the origin, where at each day you may either stay in place or move one unit in any direction, equals the number of $n$-cell standard young tableaux with $\leq 2 k+1$ rows., 2007. http://sites.math.rutgers.edu/ zeilberg/mamarim/mamarimPDF/lazy.pdf. 